\title{
Thulium fiber laser ablation of kidney stones using an automated, vibrating fiber
}

\author{
Layton A. Hall \\ David A. Gonzalez \\ Nathaniel M. Fried
}




\title{
Thulium fiber laser ablation of kidney stones using an automated, vibrating fiber
}

\author{
Layton A. Hall, ${ }^{a}$ David A. Gonzalez, ${ }^{a}$ and Nathaniel M. Fried ${ }^{a, b, c, \star}$ \\ a University of North Carolina at Charlotte, Department of Physics and Optical Science, Charlotte, North Carolina, United States \\ ${ }^{\mathrm{b}}$ Carolinas Medical Center, McKay Department of Urology, Charlotte, North Carolina, United States \\ 'Johns Hopkins Medical School, Brady Urological Institute, Baltimore, Maryland, United States
}

\begin{abstract}
Our preliminary study investigates an automated, vibrating fiber optic tip for dusting of kidney stones during thulium fiber laser (TFL) lithotripsy. A (0.75-mm diameter and 5-mm length) magnetic bead was attached to the fiber jacket, centered $2 \mathrm{~cm}$ from distal fiber tip. A solenoid was placed parallel to the fiber with a $0.5-\mathrm{mm}$ gap between solenoid and magnetic bead on fiber. The solenoid was used to create a magnetic force on the bead, inducing fiber vibration. Calibration tests for fiber motion in both air and water were performed. The ablation crater characteristics (surface area, volume, depth, and major/minor axis) of uric acid stones were measured using optical coherence tomography, after delivery of 1500 TFL pulses at $1908 \mathrm{~nm}, 33 \mathrm{~mJ}, 500 \mu \mathrm{s}$, and up to $300 \mathrm{~Hz}$, through 50-, 100-, and 150- $\mu \mathrm{m}$-core fibers. The resonant frequency was dependent on fiber diameter and rigidity, with a cutoff pivot point for optimum vibration amplitude at $4 \mathrm{~cm}$. Maximum fiber displacement is about $1 \mathrm{~mm}$ in water and $4 \mathrm{~mm}$ in air. For 50-, 100-, and 150- $\mu \mathrm{m}$-core fibers, ablated surface area averaged 1.7, 1.7, and 2.8 times greater with vibrating fiber than fixed fiber, respectively. For these fibers, ablation volume averaged 1.1, 1.5, and 1.1 times greater with vibrating fiber than fixed fiber, given a fixed energy per pulse, respectively. Our preliminary study demonstrates the functionality of an automated, vibrating fiber system for stone "dusting," with significantly larger surface area but similar ablation volumes as a fixed fiber. Future studies will focus on optimization of fiber parameters (especially displacement) and miniaturization of system components to facilitate integration into ureteroscopes. $\odot$ The Authors. Published by SPIE under a Creative Commons Attribution 4.0 Unported License. Distribution or reproduction of this work in whole or in part requires full attribution of the original publication, including its DOI. [DOI: 10 1117/1.JBO.24.3.038001]
\end{abstract}

Keywords: ablation; dusting; laser; lithotripsy; thulium; urinary stones; vibrating fiber tip.

Paper 180612R received Nov. 6, 2018; accepted for publication Mar. 11, 2019; published online Mar. 26, 2019.

\section{Introduction}

Kidney stone disease affects $\sim 10 \%$ of the U.S. population and is expected to become more prevalent, due in part to increasing obesity and diabetes. ${ }^{1,2}$ There is no cure for kidney stone disease. Medical approaches to treat kidney stones involve the use of drugs, shockwave lithotripsy (SWL), and ureteroscopy. Laser lithotripsy, involving the insertion of a flexible ureteroscope and optical fiber into the upper urinary tract, is the most effective approach to breaking up small to moderately sized kidney stones, with higher "stone-free" success rates than the established SWL method that uses ultrasound to break up stones.,

One increasingly common approach to laser ablation of kidney stones is the use of a "dusting" technique. The optical fiber is manually scanned across the stone surface, and the holmium: YAG laser emits low laser pulse energy at high pulse rates, to produce smaller fragments or stone dust for spontaneous passage through the urinary tract without the need for a stone extraction basket. This method is desirable because it removes the stone by layers and may reduce the possibility of leaving missing stone fragments inside the urinary tract. ${ }^{5-8}$ The dusting technique currently involves a handheld fiber and is thus user dependent. The potential to develop an automated method of vibrating the fiber for stone ablation is attractive, in that it may reduce user dependency and assist in optimization of the dusting technique.

\footnotetext{
*Address all correspondence to Nathaniel M. Fried, E-mail: nmfried@uncc.edu
}

Inspiration for designing a vibrating fiber for lithotripsy originated from several sources in the literature, including optical coherence tomography (OCT) probe designs and scanning fiber endoscopes. For example, a scanning OCT system has been reported, utilizing an iron, ferromagnetic bead on the fiber and a magnetic field from a solenoid to induce circular fiber motion of $\sim 3 \mathrm{~mm}$ in air., ${ }^{9,10}$ However, the 4-mm-diameter solenoid design reported in previous studies is not compatible with standard ureteroscopes, which typically have outer tip diameters of 5.2 to $8.7 \mathrm{Fr}$ ( 1.7 to $2.9 \mathrm{~mm}$ ) and working channels with inner diameters of 2.4 to $3.6 \mathrm{Fr}(0.8$ to $1.2 \mathrm{~mm}){ }^{11}$

Scanning single fiber endoscopes have also been designed, incorporating the fiber inside a piezoelectric tube that expands and contracts at the resonant frequency, creating an expanding circular motion. ${ }^{12-14}$ For this preliminary scientific study, however, the former approach (e.g., solenoid and magnetic bead on fiber) was adapted on a smaller scale to design, construct, and test a vibrating fiber for potential use in stone dusting during laser lithotripsy.

The thulium fiber laser (TFL) is currently being explored as an alternative laser source to that of the conventional holmium: YAG laser for lithotripsy. ${ }^{15,16}$ One TFL advantage is that the light originates within a small (18- to $25-\mu \mathrm{m}$ core) thuliumdoped silica fiber, so the emitted light can be coupled into smaller (50-, 100-, or 150- $\mu \mathrm{m}$-core surgical fibers) than used with the standard holmium laser $(\geq 200-\mu \mathrm{m}$ core $) .{ }^{17-19}$ These smaller fibers are more flexible and consume less cross-sectional space within the single working channel of the ureteroscope, thus yielding improved ureteroscope flexion and higher saline 
irrigation rates. These fiber characteristics (e.g., smaller diameter and less rigidity) are attractive for integration into a vibrating fiber system for potential use in laser lithotripsy in dusting mode.

\section{Theory}

From basic electromagnetic theory, ${ }^{20}$ the magnetic field in the center of a solenoid can be expressed as Eq. (1), where $\mu$ is the permeability of the core, $N$ is the number of turns on the solenoid, $I$ is the current in amperes, and $L$ is the length of solenoid:

$B=\frac{\mu N I}{L}$.

The magnetic material can be considered as a collection of magnetic dipoles. Therefore, the force can be described in Eq. (2), where $\bar{M}$ is the magnetic dipole of material, $\bar{B}$ is the magnetic field, and $\bar{F}$ is the force. A larger magnetic dipole and a larger magnetic field produce a greater force:

$\bar{F}=\nabla(\bar{M} \cdot \bar{B})$

Application of a magnetic field alone will not achieve the motion desired in this study. A periodic signal needs to be applied to have a "vibrating" fiber.

The fiber vibration (based on pivot point and medium) can be modeled as a simple harmonic oscillator with damping, ${ }^{10}$ incorporating damping across the entire fiber length, as described by

$$
\frac{d^{2} x}{d t^{2}}+\gamma \frac{d x}{d t}+\omega_{0}^{2} x=a_{0} e^{-i \omega t}
$$

where $a_{0}$ is the applied force divided by mass of object. In addition, $\omega_{0}$ is the resonant frequency of the system and $\omega$ is the driving frequency. The steady-state solution is given as

$$
x(t)=\frac{a_{0} \cos (\omega t-\varphi)}{\sqrt{\left(\omega_{0}^{2}-\omega^{2}\right)^{2}+(\gamma \omega)^{2}}} .
$$

This equation provides a method of understanding the damping effects. In practical application, the amplitude at the resonant frequency changes, damping the resulting motion. This is an unavoidable but also undesirable result, given the goal of producing a vibrating fiber.

The model above is oversimplified because it does not take into account the entire fiber or lever. A more accurate model is the uniformly damped Euler-Bernoulli beam equation or the following equation:

$E I \frac{\partial^{4} w(x, t)}{\partial x^{4}}+A \rho \frac{\partial^{2} w(x, t)}{\partial t^{2}}+\gamma \frac{\partial w(x, t)}{\partial t}=q(x, t)$,

where $E$ is the Young's modulus, $I$ is the area moment of inertia, $A$ is the cross-sectional area, $\rho$ is the mass density of the fiber, $\gamma$ is the damping, $w(x, t)$ is the displacement of the fiber, and $q(x, t)$ is the force being applied to the fiber. Some interesting characteristics of this differential equation, which will be observed in the calibration study, are that the maximum displacement at the end of the cantilever, $w_{\max }$, is described by Eq. (6), if the force is applied to the free end of the fiber with no damping $w_{\max }=\frac{q_{\max } L^{3}}{3 E I}$.

In addition, the first-order resonant frequency can be determined for this simple one-dimensional model, provided as

$\omega_{1}=\frac{3.5161}{L^{2}} \sqrt{\frac{E I}{\rho}}$.

Just for reference, Young's modulus and the area moment of inertia can also be defined in Eqs. (8) and (9), where $\sigma$ is the tensile stress, $\varepsilon$ is the strain, $m$ is the mass, and $r$ is the radius of the cylinder. ${ }^{21}$ In this application, Young's modulus will remain constant, but the area moment of inertia will vary as the radius of the fiber to the fourth power.

$E=\frac{\sigma(\varepsilon)}{\varepsilon}$,
$I_{x x}=\iint x^{2} \mathrm{~d} x \mathrm{~d} y \propto r^{4}$.

Finally, the stiffness of a material can be measured by the force applied over the amount displaced, as described in Eq. (10), where $A$ is the cross-sectional area, $E$ is the Young's modulus, and $L$ is the length of material:

$k=\frac{F}{d}=\frac{A E}{L}$.

For the relevant case of an optical fiber in different environments (e.g., air and water), the drag force, $F$, can be defined by Eq. (11), where $\rho$ is the density of the medium, $A$ is the crosssectional area, $v$ is the velocity, and $\beta$ is the damping coefficient. As an example, the $\beta$ value for a cylinder is $\sim 1.15$. The density for air and water is $\sim 1.3$ and $998 \mathrm{~kg} / \mathrm{m}^{3}$, respectively. In terms of the drag force, the large difference in densities is partially negated by the lower velocity in water (damped case) than in air:

$F=0.5 \rho v^{2} \beta A$.

\section{Methods}

\subsection{Vibrating Optical Fiber Setup}

To maximize the force produced on the fiber, there are a few trends that can be observed from Eqs. (1) and (2). First, the force produced is linearly dependent on the magnitude of the magnetic field. To produce a strong magnetic field, a high permeability material was used for the solenoid core, consisting of a ferrite rod of 1.0-mm diameter and 7.5-mm length with ferrite material and an initial permeability of $2300 \mathrm{H} / \mathrm{m}$ (R78-004-039, Amidon Corporation, Costa Mesa, California). In addition, the density of turns [Eq. (1)] for the solenoid should be maximized to induce a large force. Therefore, the solenoid was tightly and symmetrically wrapped with a 36 gauge wire $(0.127-\mathrm{mm}$ diameter), 150 times across the 7.5-mm length.

The magnetic bead composition determines the magnetization [shown in Eq. (2)], which directly affects the magnitude of the force. The magnetic bead was constructed out of 300nm-diameter synthetic black iron oxide $\left(\mathrm{Fe}_{3} \mathrm{O}_{4}\right)$ particles (Alpha Chemicals, Cape Girardeau, Missouri). The particles 
Table 1 Specifications of optical fibers tested.

\begin{tabular}{lcccc}
\hline Manufacturer & Part No. & $\begin{array}{c}\text { Core } \\
(\mu \mathrm{m})\end{array}$ & $\begin{array}{c}\text { Cladding } \\
(\mu \mathrm{m})\end{array}$ & $\begin{array}{c}\text { Coating } \\
(\mu \mathrm{m})\end{array}$ \\
\hline $\begin{array}{l}\text { Polymicro } \\
\text { Technologies }\end{array}$ & 1068000345 & $50 \pm 2$ & $70 \pm 2$ & $85 \pm 3$ \\
$\begin{array}{l}\text { Polymicro } \\
\text { Technologies }\end{array}$ & 1068000071 & $100 \pm 3$ & $140 \pm 3$ & $170 \pm 5$ \\
$\begin{array}{l}\text { Polymicro } \\
\text { Technologies }\end{array}$ & 1068000059 & $150 \pm 3$ & $180 \pm 3$ & $200 \pm 5$ \\
\hline
\end{tabular}

were mixed with an adhesive (B00OL1ZF0E, Craft Smart) with concentration of two parts iron oxide particles and one part adhesive. The bead of $0.75 \mathrm{~mm}$ in diameter and $5 \mathrm{~mm}$ in length was then attached $2 \mathrm{~cm}$ from the distal fiber tip. The distance or gap between the parallel oriented solenoid and magnetic bead on the fiber was fixed at $0.5 \mathrm{~mm}$ for all of the studies.

Fiber motion was induced by applying an AC voltage to the solenoid using a signal generator (3312A, Agilent Technologies, Santa Clara, California) and 1-A output current, dual power operation amplifier (TCA072, ON Semiconductors, Newburyport, Massachusetts). Although it was possible to induce circular motion of the fiber, this motion was more difficult to achieve due to the high damping conditions (e.g., water medium) typically encountered during lithotripsy. Therefore, all of the calibration and stone ablation studies described below utilized induction of linear motion only. The list of optical fiber specifications is shown in Table 1, and the vibrating fiber experimental setup is shown in Fig. 1.

\subsection{System Characterization}

To characterize fiber motion, frequency response versus fiber tip displacement and pivot point versus fiber tip displacement in both air and water mediums were measured and repeated $(n=3$ for each set of parameters). A CMOS camera (DCC1645C-HQ, Thorlabs, Newton, New Jersey) was used to image the vibrating fiber tip, and a common blob analysis technique in MATLAB
(MathWorks, Natick, Massachusetts) was used to measure fiber displacement. A pixel-to-millimeter ratio was determined to find the fiber displacement.

TFL lithotripsy experiments using uric acid (UA) stone samples with the vibrating fiber setup were conducted. Stone samples were prepared using a wet saw to produce a smooth surface on one face. The stone was then ablated with a fixed number of 1500 laser pulses. An OCT system (CAL110C1, Calisto, Thorlabs, Newton, New Jersey) was used for three-dimensional imaging of the stone ablation crater. OCT characterization of stone ablation crater dimensions is a common technique in the field. ${ }^{19,22-24}$ The OCT system operated at a center wavelength of $930 \mathrm{~nm}$, with spectral bandwidth of $100 \mathrm{~nm}$, and axial and lateral resolutions in air of 7 and $8 \mu \mathrm{m}$, respectively. Stone ablation crater volume, surface area, and depth were calculated from OCT data, as well as major and minor axis ratio, to determine ablation characteristics and analyze whether automated vibration produces a dusting-like ablation technique. The OCT system did not contain a volume or surface area measurement software feature. Therefore, the OCT surfaces were imported for analysis into MATLAB to characterize the crater. Numerical methods of integration were used to obtain the area and volume, given a user-defined reference surface (e.g., the polished flat surface) at the entrance of the craters. The major and minor axes were measured by pixel count.

The first calibration study focused on the fundamental motion of the fibers. Input voltage and displacement were plotted for 50-, 100-, and $150-\mu \mathrm{m}$-core fibers. (Fibers that were $>200-\mu \mathrm{m}$ core were considered during initial studies as well. However, after calibration results, no significant fiber displacement was observed.) Figure 2 shows that the theoretical linear relationship between input current and displacement, suggested by Eqs. (1) and (2), is confirmed during experimental calibration of the system. These calibration curves also demonstrate a simple way to control fiber displacement based on input voltage. The curve fitting shown in Fig. 2 used a least squared sum methodology in MATLAB to fit the data to a linear curve.

The second calibration study measured dependence of fiber displacement on pivot point to determine optimum distance. In Fig. 3, as the pivot point increases, displacement increases as well, as expected from Eq. (6). However, there is a saturation

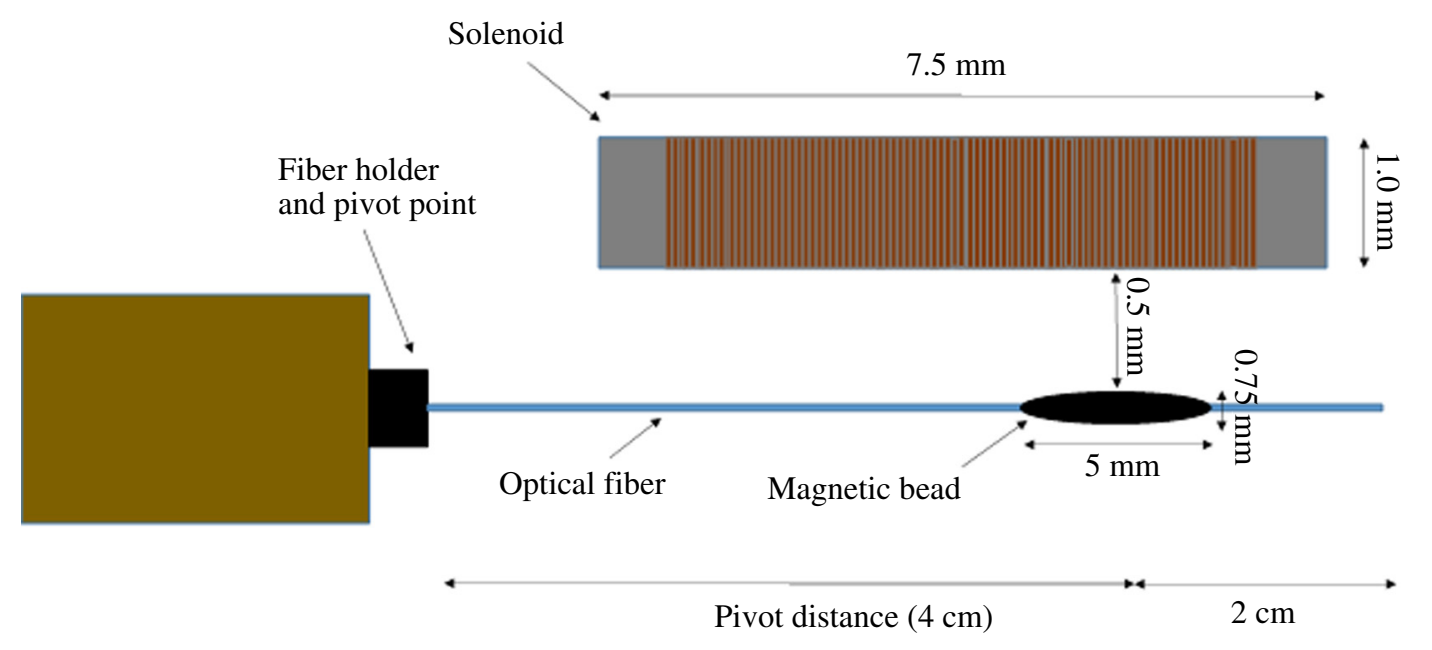

Fig. 1 Construction of vibrating fiber, consisting of a ferrite core solenoid with 36 gauge wire wrapping, optical fiber with magnetic bead attached, and a fiber holder, acting as a pivot point for the motion. For system calibration and laser stone ablation studies, a pivot distance of $4 \mathrm{~cm}$ was used. 


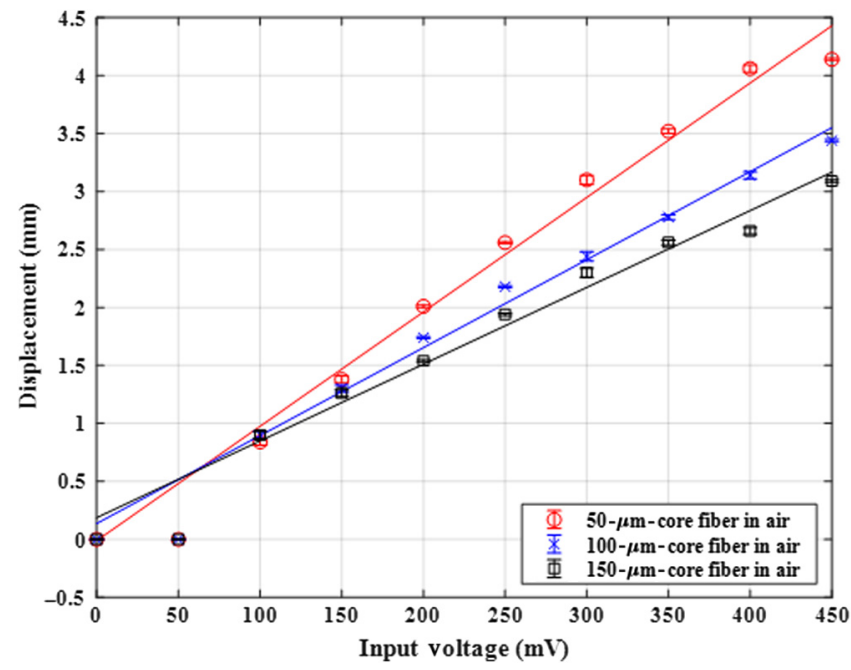

Fig. 2 Calibration curves at a 4-cm pivot point for 50-, 100-, and $150-\mu \mathrm{m}$-core fibers in air, showing a direct linear relationship between current and force [Eqs. (1) and (2)]. A standard linear fit was applied, yielding $R^{2}$ values of $0.96(50-\mu \mathrm{m}$-core fiber $), 0.99(100-\mu \mathrm{m}$-core fiber), and 0.99 (150- $\mu \mathrm{m}$-core fiber).

point at which the maximum displacement is achieved, due to limitations imposed by the experimental setup (e.g., confined space for vibration given the fiber and solenoid were oriented parallel to each other and in close proximity). For practical use, the pivot point should be selected based on values that are near the edge of these maxima. The pivot point versus maximum displacement curves is interesting in nature due to their odd shape. From Eq. (6), it is observed that the beam will follow an $L^{3}$ model scaled by Young's modulus and area moment of inertia. Note, Young's modulus is constant for a given material [Eq. (7)] and the area moment of inertia on an elementary level is proportional to the radius to the fourth of a cylinder [Eq. (8)]. This explains the nature of the curve and why the $50-\mu \mathrm{m}$-core fiber produces a steeper slope than the 100- and 150- $\mu \mathrm{m}$-core

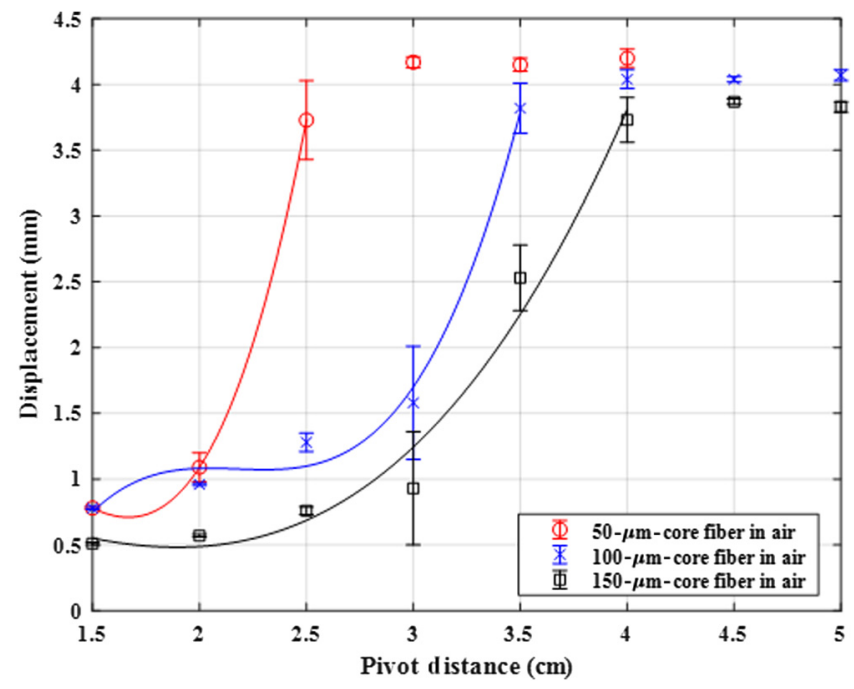

Fig. 3 Calibration curves for 50-, 100-, and 150- $\mu$ m-core fibers, showing the relationship between the location of the pivot point and fiber displacement. A third-order polynomial fit was applied, yielding $R^{2}$ values of $0.99(50-\mu \mathrm{m}$-core fiber), 0.98 (100- $\mu \mathrm{m}$-core fiber), and 0.97 (150- $\mu \mathrm{m}$-core fiber).

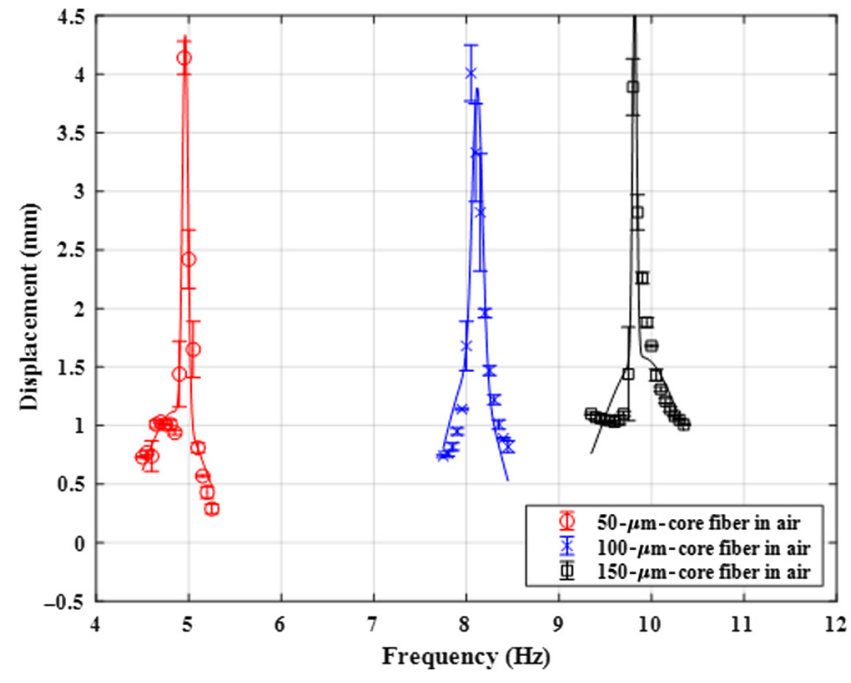

Fig. 4 Frequency response in air at a pivot point of $4 \mathrm{~cm}$ for 50-, 100-, and $150-\mu \mathrm{m}$-core fibers. A bimodal Gaussian fit was applied, yielding $R^{2}$ values of 0.95 (50- $\mu \mathrm{m}$-core fiber), 0.93 (100- $\mu \mathrm{m}$-core fiber), and 0.90 (150- $\mu \mathrm{m}$-core fiber).

fibers since the outer diameter of the $50-\mu \mathrm{m}$ fiber (Table 1 ) is less than half the 100- and $150-\mu \mathrm{m}$-core fiber outer diameters. The theory also explains the similar characteristics of 100- and $150-\mu \mathrm{m}$-core fibers since their fiber outer diameters differ by only $30 \mu \mathrm{m}$. To perform the fit in Fig. 3, the "polyfit" function was used in MATLAB. Since the theory suggests the displacement should be an $L$ to the third curve, it was chosen to fit the data to a third-order polynomial up until the saturation point for each fiber.

Finally, one of the defining characteristics of this system is the frequency response and how the damping of the surrounding medium affects the system. To characterize frequency response, the fiber was placed in air and water at a pivot point of $4 \mathrm{~cm}$ and displacement of the fiber was measured as a function of frequency (Figs. 4 and 5). Initial tests were performed in both saline and water, but no difference was observed, so water was

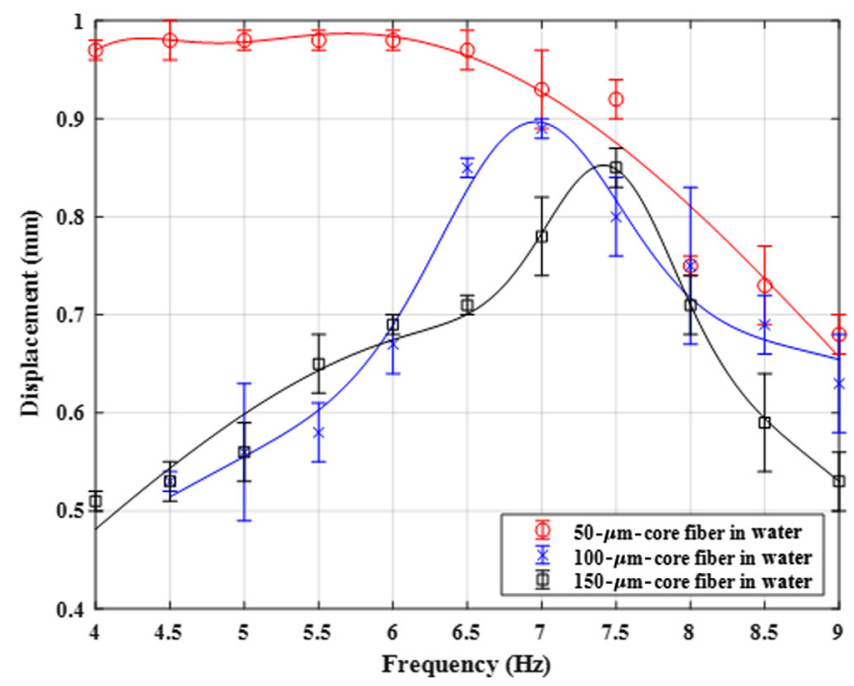

Fig. 5 Frequency response in water at a pivot point of $4 \mathrm{~cm}$ for $50-$, $100-$, and $150-\mu \mathrm{m}$-core fibers. A bimodal Gaussian fit was applied, yielding $R^{2}$ values of 0.95 (50- $\mu \mathrm{m}$-core fiber), $0.97(100-\mu \mathrm{m}$-core fiber), and 0.97 (150- $\mu \mathrm{m}$-core fiber). 
used for the experiments. There was a decrease in maximum fiber displacement in air $(\sim 4 \mathrm{~mm})$ versus water $(\sim 1 \mathrm{~mm})$, which may be explained by the damping effect of the water medium [Eqs. (3), (4), and (11)]. In addition to this observation, Eq. (7) explains the characteristics of the resonant frequency of a cantilever and the experimental results. Since the length $(L)$ remains constant for all fibers tested, the only dependence is on Young's modulus (constant for a given material), area moment of inertia (varies with diameter), and mass density (which in principle should remain constant for all fibers across the entire length of each fiber). Therefore, as stated earlier, since the 50- $\mu \mathrm{m}$-core fiber has a lower area moment of inertia [Eq. (8)] compared to the 100 - and $150-\mu \mathrm{m}$-core fiber, the $50-\mu \mathrm{m}$-core

Table $2 \operatorname{TFL}(\lambda=1908 \mathrm{~nm})$ parameters.

\begin{tabular}{lccccc}
\hline $\begin{array}{l}\text { Fiber } \\
\text { core }(\mu \mathrm{m})\end{array}$ & $\begin{array}{c}\text { Pulse } \\
\text { energy } \\
(\mathrm{mJ})\end{array}$ & $\begin{array}{c}\text { Pulse } \\
\text { duration } \\
(\mu \mathrm{s})\end{array}$ & $\begin{array}{c}\text { Pulse rate } \\
(\mathrm{Hz})\end{array}$ & $\begin{array}{c}\text { Average } \\
\text { power }(\mathrm{W})\end{array}$ & $\begin{array}{c}\text { Laser } \\
\text { pulses } \\
(\#)\end{array}$ \\
\hline 50 & 33 & 500 & 50 & 1.65 & 1500 \\
100 & 33 & 500 & 300 & 10.3 & 1500 \\
150 & 33 & 500 & 300 & 10.3 & 1500 \\
\hline
\end{tabular}

fiber also has a lower resonant frequency than the other two fibers. In addition, the 100- and 150- $\mu \mathrm{m}$-core fibers have similar resonant frequencies since they have similar diameters for their area moment of inertia. To perform the optimum fit (for Figs. 4 and 5), the "fit" function was used in MATLAB using a bimodal Gaussian as the base function due to the overall Gaussian nature of the curves and the generic nature of the Gaussian.

\subsection{Laser Parameters}

A 100-W, continuous-wave, TFL (TLR 100-1908, IPG Photonics, Oxford, Massachusetts) with a wavelength of $1908 \mathrm{~nm}$ was used in the stone ablation studies. A 25-mm-focallength, AR-coated, plano-convex lens focused the 5.5-mmdiameter laser beam from the built-in collimator to a spot diameter of $\sim 25 \mu \mathrm{m}\left(1 / e^{2}\right)$ for coupling into separate low-OH, silica optical fibers of 50-, 100-, or 150- $\mu \mathrm{m}$-core diameters (Table 1). The laser was electronically modulated with a function generator (DS345, Stanford Research Systems, Sunnyvale, California) to produce an energy per pulse of $33 \mathrm{~mJ}$, pulse duration of $500 \mu \mathrm{s}$, and pulse rates up to $300 \mathrm{~Hz}$ (Table 2). However, power delivery through the $50-\mu \mathrm{m}$-core fiber was limited due to difficulties in alignment of the small fiber core. The laser parameters are summarized in Table 2.
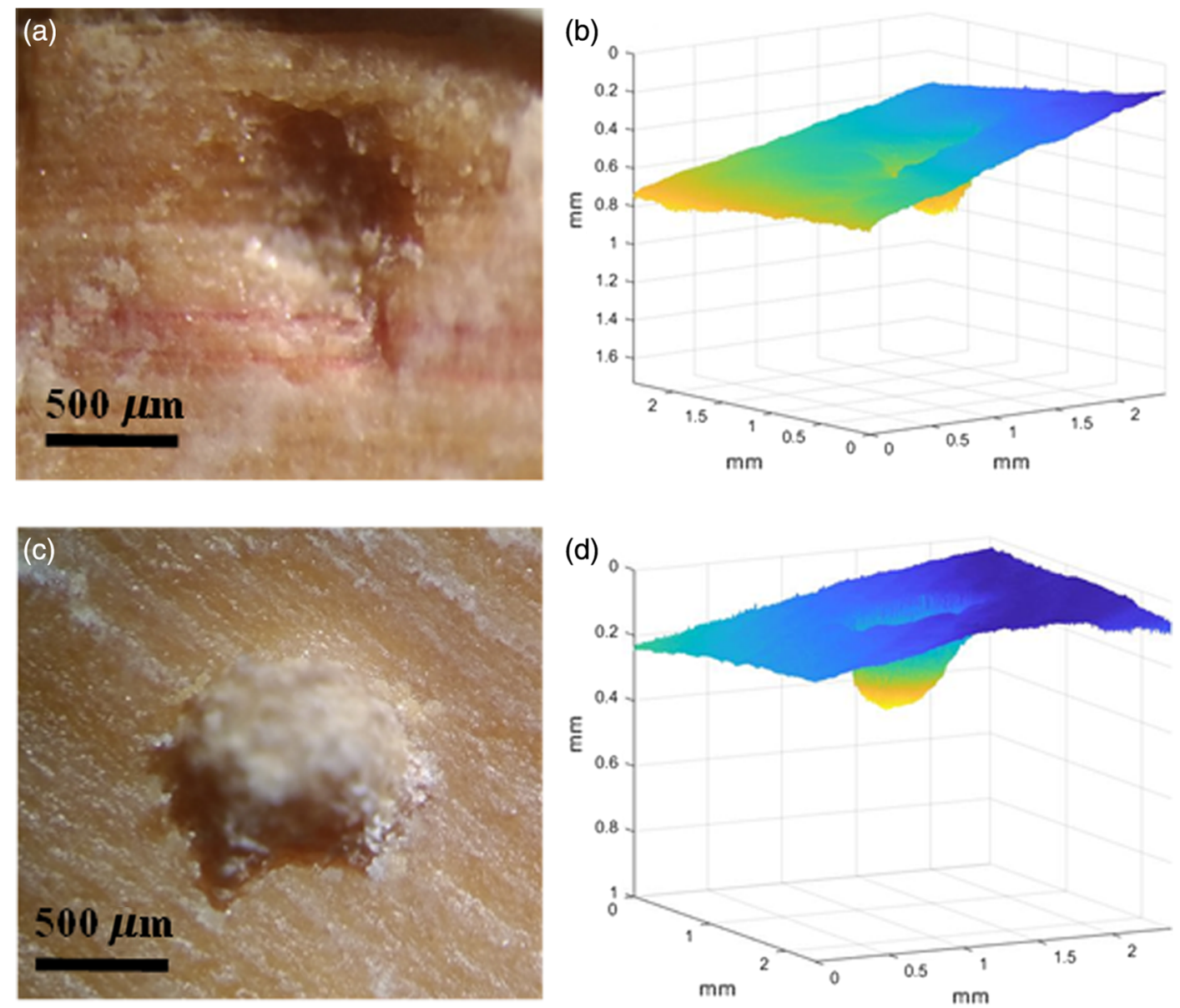

Fig. 6 Comparison between (a, b) fixed fiber and (c, d) vibrating fiber ablation for $50-\mu \mathrm{m}$-core fiber. Images (b, d) are OCT image reconstructions in MATLAB to determine cross-sectional area, volume, and axis length. 


\subsection{Statistical Analysis}

A student's $t$-test was performed comparing ablation crater dimensions (volume, surface area, depth, major axis, and minor axis) between vibrating and fixed fibers for each fiber size (50-, 100-, and 150- $\mu \mathrm{m}$ core). Each calibration study was performed three times $(n=3)$, with the data points and error bars in the calibration figures representing the mean and standard deviation, respectively. Each stone ablation study was performed five times $(n=5)$. The mean and standard deviation for the stone ablation crater characteristics are also provided in the tables. A value of $p<0.05$ was considered to be statistically significant.

\section{Results}

A comparison of TFL stone ablation between vibrating and fixed fibers was conducted. For the vibrating fiber ablation experiments, a voltage setting of $450 \mathrm{mV}$ (corresponding to a maximum allowable current of $200 \mathrm{~mA}$ for the wire in the solenoid) and a fixed pivot point of $4 \mathrm{~cm}$ was used, based on the results of the calibration studies (Figs. 2 and 3). The frequency $(\mathrm{Hz})$ setting was different for each fiber diameter and was chosen based on the individual resonant frequencies in water measured during the calibration studies (Fig. 5). The distal fiber tip was placed $500 \mu \mathrm{m}$ from the stone surface in noncontact mode for each study and the edge of the magnetic bead was

Table 3 Vibrating versus fixed fiber operation mode results for $50-\mu \mathrm{m}$ core fiber $(n=5)$.

\begin{tabular}{|c|c|c|c|c|c|c|c|}
\hline & Stone & Volume $\left(\mathrm{mm}^{3}\right)$ & Surface area $\left(\mathrm{mm}^{2}\right)$ & Depth (mm) & Major axis (mm) & Minor axis (mm) & Ratio \\
\hline \multirow[t]{2}{*}{ Fixed fiber } & Mean & 0.08 & 0.27 & 0.26 & 0.83 & 0.45 & 1.84 \\
\hline & St. Dev. & 0.01 & 0.05 & 0.06 & 0.13 & 0.08 & - \\
\hline \multirow[t]{2}{*}{ Vibrating fiber } & Mean & 0.09 & 0.46 & 0.21 & 0.88 & 0.67 & 1.31 \\
\hline & St. Dev. & 0.02 & 0.04 & 0.06 & 0.03 & 0.05 & - \\
\hline Statistics & $P$-value & 0.17 & 0.0002 & 0.22 & 0.42 & 0.0008 & - \\
\hline
\end{tabular}
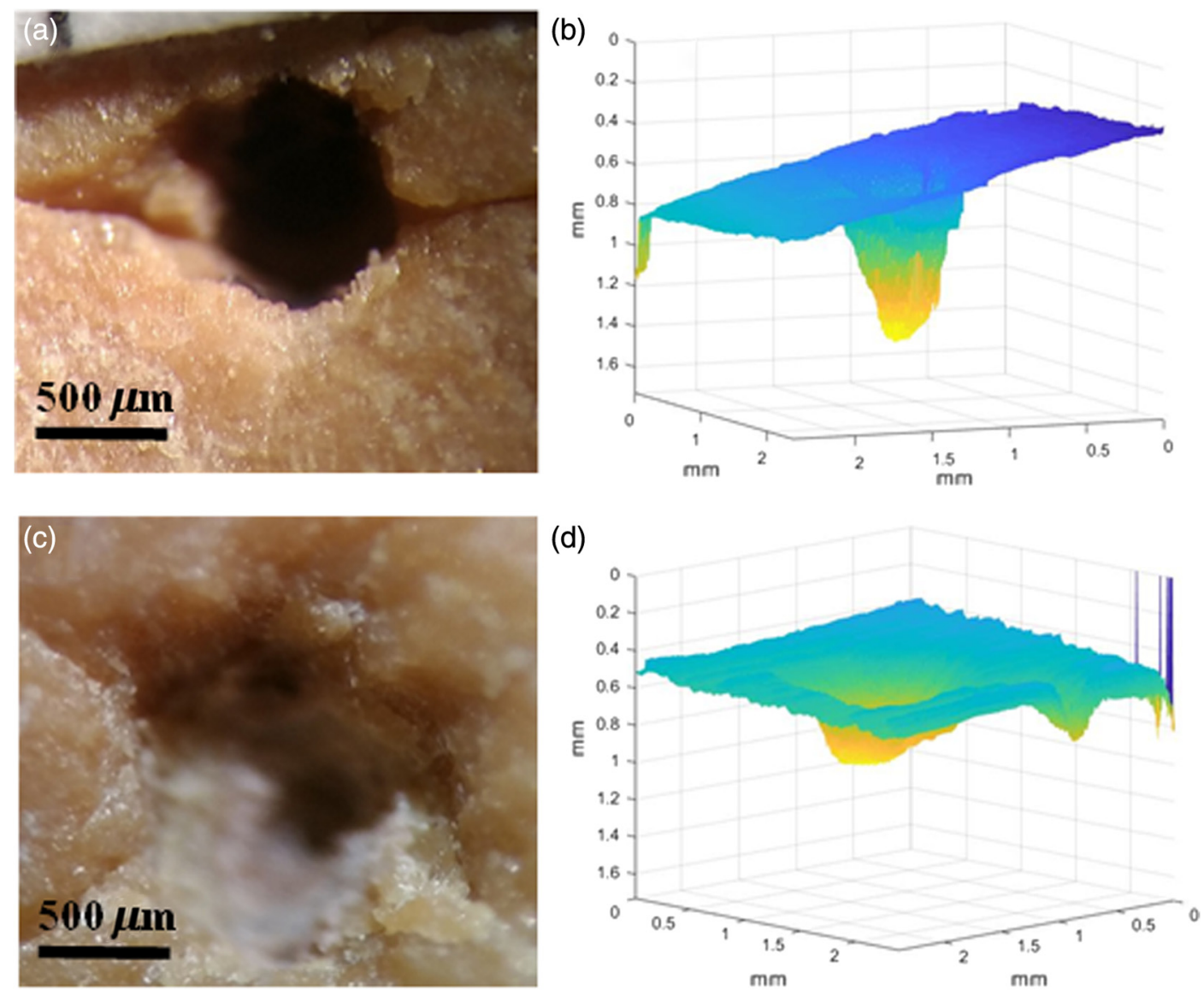

Fig. 7 Comparison between (a, b) fixed fiber and (c, d) vibrating fiber ablation for 100- $\mu \mathrm{m}$-core fiber. Images (b, d) are OCT image reconstructions in MATLAB to determine cross-sectional area, volume, and axis length. 
placed $500 \mu \mathrm{m}$ away from the edge of the solenoid. The stone was then ablated with 1500 laser pulses (Table 2).

The data for the 50- $\mu \mathrm{m}$ core fiber are shown in Fig. 6 and Table 3. An interesting result is the shape of the craters. The fixed fiber produced elliptical crater surfaces, as quantified by the ratio of the major to minor axis listed in Table 3 . However, the vibrating fiber produced an ablation crater surface with a more circular profile, as observed in Figs. 6(c) and 6(d). The volumes of the vibrating and fixed fiber ablation craters were similar $(p=0.17)$. The surface area of the ablation crater with the vibrating fiber was on average 1.7 times greater than for the fixed fiber $(p=0.0002)$.
The results for the $100-\mu \mathrm{m}$ fiber are shown in Fig. 7 and Table 4 . There is a noticeable difference between the vibrating and fixed fiber ablation craters. The fixed fiber produced a deeper crater by about a factor of 2.8 , consistent with a more "drilling" approach to ablation. However, the vibrating fiber produced on average 1.7 times larger ablation surface area $(p=0.0006)$. The vibrating method also produced a more elliptical crater than the fixed fiber, having a major-to-minor axis ratio of 1.4 times larger. Note, there were cracks observed during all of the $100-\mu \mathrm{m}$ fiber studies involving fixed fiber ablation [Fig. 7(a)], which could be due in part to the deeper ablation crater, and also indicative of a more "fragmentation"-like

Table 4 Vibrating versus fixed fiber operation mode results for $100-\mu \mathrm{m}$ core fiber $(n=5)$.

\begin{tabular}{|c|c|c|c|c|c|c|c|}
\hline & Stone & Volume $\left(\mathrm{mm}^{3}\right)$ & Surface area $\left(\mathrm{mm}^{2}\right)$ & Depth (mm) & Major axis (mm) & Minor axis (mm) & Ratio \\
\hline \multirow[t]{2}{*}{ Fixed fiber } & Mean & 0.22 & 0.43 & 0.91 & 0.82 & 0.72 & 1.14 \\
\hline & St. Dev. & 0.03 & 0.02 & 0.03 & 0.03 & 0.06 & - \\
\hline \multirow[t]{2}{*}{ Vibrating fiber } & Mean & 0.33 & 0.75 & 0.32 & 1.29 & 0.78 & 1.65 \\
\hline & St. Dev. & 0.17 & 0.13 & 0.06 & 0.12 & 0.10 & - \\
\hline Statistics & $P$-value & 0.20 & 0.0006 & $5 \times 10^{-8}$ & 0.0004 & 0.28 & - \\
\hline
\end{tabular}
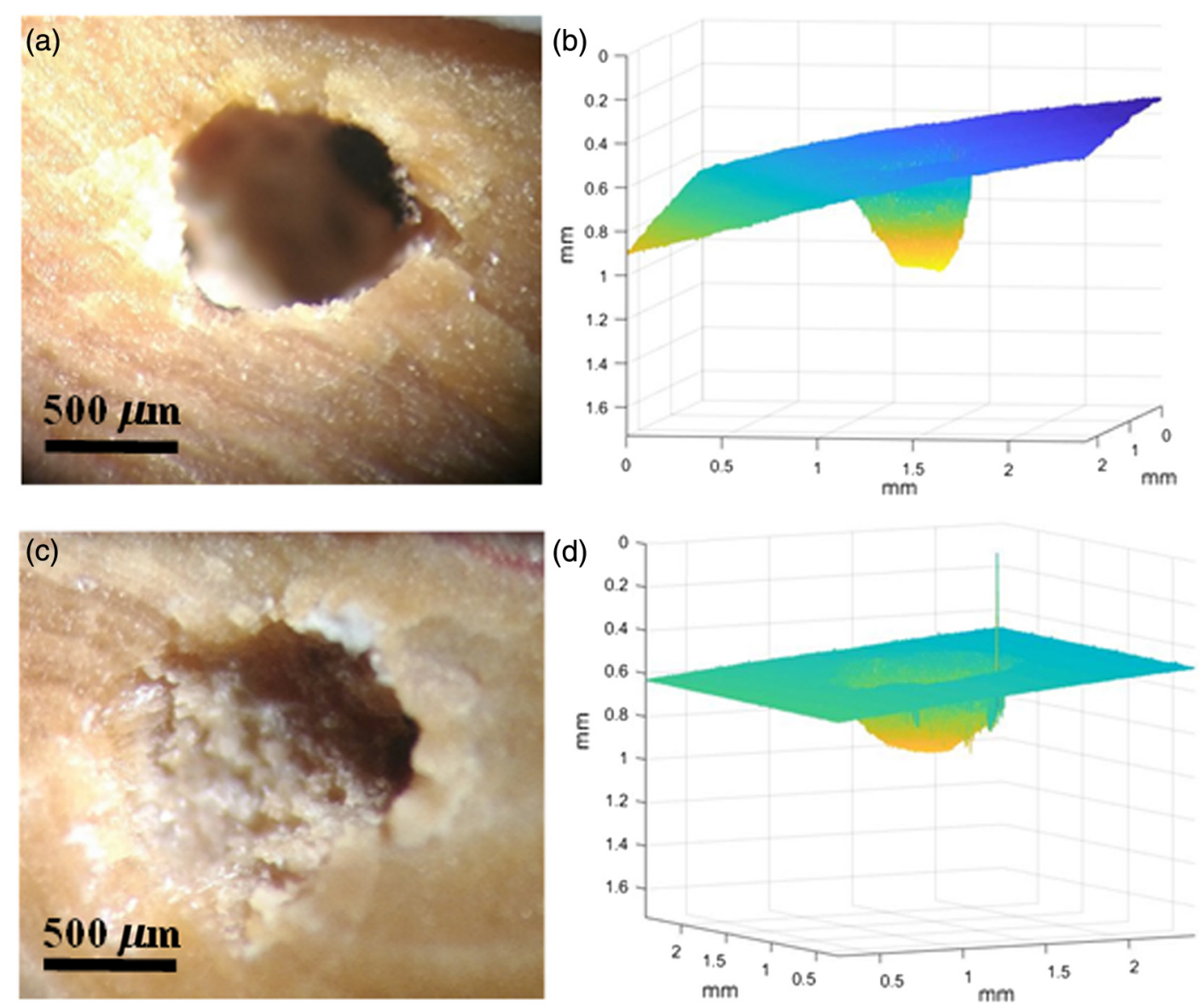

Fig. 8 Comparison between (a, b) fixed fiber and (c, d) vibrating fiber ablation for 150- $\mu$ m-core fiber. Images $(b, d)$ represent the OCT imaging reconstruction in MATLAB to determine cross-sectional area, volume, and axis length. 
Table 5 Vibrating versus fixed fiber operation mode results for $150-\mu \mathrm{m}$ core fiber $(n=5)$.

\begin{tabular}{|c|c|c|c|c|c|c|c|}
\hline & Stone & Volume $\left(\mathrm{mm}^{3}\right)$ & Surface area $\left(\mathrm{mm}^{2}\right)$ & Depth (mm) & Major axis (mm) & Minor axis $(\mathrm{mm})$ & Ratio \\
\hline \multirow[t]{2}{*}{ Fixed fiber } & Mean & 0.21 & 0.24 & 0.89 & 0.64 & 0.48 & 1.33 \\
\hline & St. Dev. & 0.01 & 0.09 & 0.03 & 0.16 & 0.08 & - \\
\hline \multirow[t]{2}{*}{ Vibrating fiber } & Mean & 0.23 & 0.67 & 0.37 & 1.17 & 0.74 & 1.58 \\
\hline & St. Dev. & 0.07 & 0.23 & 0.04 & 0.19 & 0.19 & - \\
\hline Statistics & $P$-value & 0.54 & 0.005 & $1 \times 10^{-8}$ & 0.002 & 0.02 & - \\
\hline
\end{tabular}

approach with a fixed fiber as compared to a "dusting" approach with the vibrating fiber, as anticipated.

The results for the 150- $\mu$ m-core fiber are shown in Fig. 8 and Table 5, with trends similar to the other fiber sizes. The volume removed from each of the stones was similar for the vibrating and fixed fibers $(p=0.54)$. However, the surface area covered by the vibrating fiber was a factor of 2.8 times greater than for the fixed fiber $(p=0.005)$. In addition, as shown in Fig. 8 and supported by data in Table 5, the ratio of the major and minor axis for the vibrating fiber produces a more elliptical crater (as defined by a greater value for the ratio of major to minor axis), whereas the fixed fiber produced a more circular crater.

\section{Discussion}

\subsection{System Calibration}

Each of the fiber sizes completed a series of calibration tests $(n=3)$ to characterize the complex nature of the motion. The first test results (Fig. 2) show the multiple characteristics of the maximum fiber displacement, demonstrating the linear relationship between current input and force on magnetic bead, as described in Eqs. (1) and (2). Fiber displacement decreases with increasing fiber outer diameter and fiber stiffness. This can also be explained by the increase in Young's modulus and moment of inertia increase with increasing fiber diameter. $^{25,26}$

The dependence of fiber displacement on the pivot point distance was also demonstrated (Fig. 3), with an increase in pivot point also resulting in an increase in fiber displacement until saturation after a pivot point distance of $2.5 \mathrm{~mm}$ for $50-\mu \mathrm{m}$ core fiber and $4 \mathrm{~mm}$ for the $100-$ and $150-\mu \mathrm{m}$-core fibers. For the implementation of this device, the pivot point should be placed at these critical points to minimize the length of the pivot point and achieve maximum displacement.

The next calibration study represented displacement as a function of frequency for different fiber diameters in both air and water (Figs. 4 and 5). The first major difference is the sharpness of the peaks in air. In air, the damping coefficient is relatively small. According to the simplified model of Eq. (4), as damping decreases, the peaks become sharper (Fig. 9). Note, to demonstrate the effects of the sharpness of the peaks and change in resonant frequencies, the graphs were normalized since a large damping effect would make the maximum amplitude much less than the small damping amplitude. In addition, the water calibration curves have a general inflection similar to the theoretical model of Eq. (4) and the damped case in Fig. 9. The curves are not identical due to the oversimplified model used in Eq. (4) but show the general agreement between theory and measured results.

The error bars observed in the calibration data primarily occur in regions where the data are changing rapidly (e.g., at resonant frequencies in air or the rapid increase in displacement based on pivot point). However, if the system is stable and in a steady-state condition, the error bars decrease significantly. Nevertheless, for procedures performed in the clinic, where the fiber is in a submerged water environment (highly damped media), the resonant curves are widened, leaving a significant region to find the optimum frequency, as observed in Fig. 5.

In addition to the sharpness of the curves, resonant frequencies change based on fiber diameter (and stiffness) and the damping factor that is observed. Change in resonant frequency can be explained in an analog way as a spring change in resonant frequency based on the spring stiffness. Similarly, the fiber's resonant frequencies change with the fiber diameter and stiffness, increasing as the fiber becomes more rigid. The resonant frequency can also change as a function of damping [Eq. (4) and Fig. 9]. As damping (e.g., air to water) increases, the resonant frequency shifts to a lower frequency. Overall, this trend explains the frequency response of the fibers in the experiments. In addition, optimum conditions were determined to be at a pivot

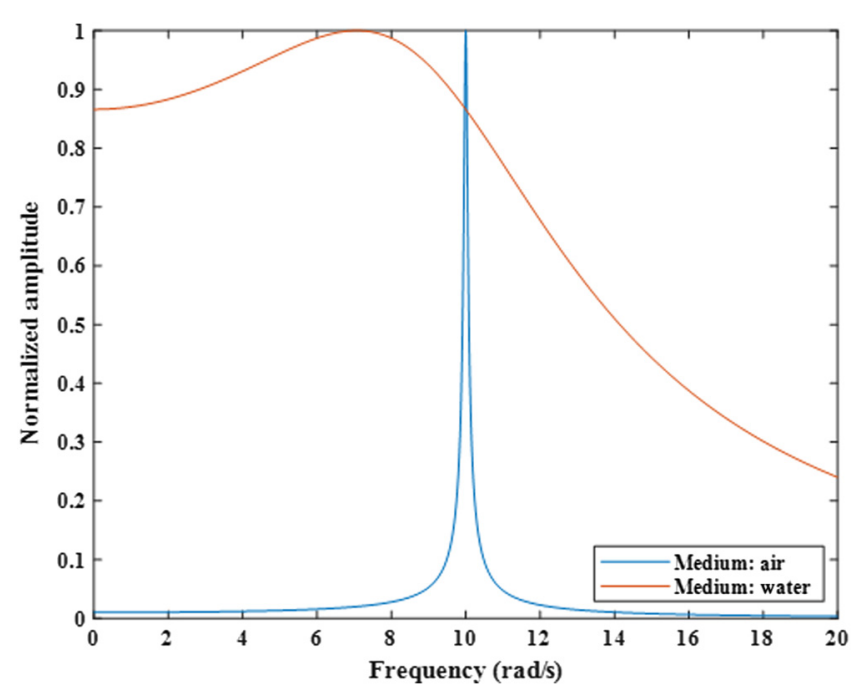

Fig. 9 The fiber displacement curve as a function of medium (air and water) at a frequency $\left(\omega_{0}\right)$ of $5 \mathrm{~Hz}$. The curves are plotted on a normalized amplitude scale to the maximum value of each curve. The plot demonstrates how damping broadens the displacement curves and the resonant frequency according to Eq. (4). 
point distance of $4 \mathrm{~cm}$ and frequency of $5 \mathrm{~Hz}$ in water across all fibers to keep driving settings consistent.

\subsection{Stone Ablation}

For the UA stone ablation studies, there was also normal motion of the distal fiber tip, for both fixed and vibrating fibers, during laser emission, presumably due to laser-induced vapor bubbles producing an impulse effect on the fiber. This laser-induced fiber motion was more prevalent in the smaller $50-\mu \mathrm{m}$-core fiber than the 100- and 150- $\mu \mathrm{m}$-core fibers, consistent with previous observations. ${ }^{18}$ Despite this normal motion, the solenoid was able to produce a sufficient force to enlarge the crater surface area produced by the fiber.

For the $100-\mu \mathrm{m}$-core fibers, there was a significant difference in size of the ablation crater surface areas (Fig. 7). The ratio of the major and minor axis increased by $46 \%$, and the surface area covered by the vibrating fiber increased by an average factor of 1.7. However, the ablation crater depth was 2.8 times greater for the fixed fiber. This result can be explained by the fiber moving over a greater surface area for the same period of time, thus translating into a decreased dwell time and hence ablation depth at any given point. The volume removed was similar between vibrating and fixed fibers, consistent with previous studies, which reported that ablation rate is independent of fiber diameter for a similar pulse energy used. ${ }^{27}$

The $150-\mu \mathrm{m}$-core fiber had similar results with ratio of major to minor axis of the ablation craters increasing by an average of $19 \%$, a surface area by an average factor of 2.8 , and similar ablation volumes between vibrating and fixed fibers $(p=0.54)$. While 100 - and $150-\mu \mathrm{m}$-core fibers produced similar results, the $50-\mu \mathrm{m}$-core fiber was slightly different. The first noticeable difference was the shape of the ablation craters (Fig. 6). The vibrating fiber produced a more circular crater with a major and minor axis ratio of 1.31 on average, whereas the fixed fiber produced an average ratio of 1.84 , or more elliptical motion, contrary to what was predicted. The most probable explanation is that the solenoid produced a force perpendicular and similar in magnitude to that of the laser-induced vapor bubbles. This may explain how the vibrating fiber would create a more circular crater.

As previously shown, the Young's modulus and area moment of inertia determine stiffness of a solid material. Since the area moment of inertia is (on an elementary level) proportional to the radius of the cylinder to the fourth, this shows a direct dependence on the fiber size (e.g., as fiber diameter increases, the more stiff it will be, given the material constant remains the same). An interesting result of this experiment is the 100 - and $150-\mu \mathrm{m}$-core fibers produced similar surface area and displacements, but the $50-\mu \mathrm{m}$-core fiber produced less. From analysis of the stone ablation videos, it was observed that fiber tip vibration was not only produced by the solenoid induced motion but also from laser emission as well. It is speculated that the relatively small amplitude of fiber motion allowed the laser-induced vapor bubble to induce another force, thus providing torque to increase fiber vibration, in turn, increasing the fiber motion during stone ablation. Since the $50-\mu \mathrm{m}$ fiber studies were conducted at a lower pulse rate and average power than the 100 - and $150-\mu \mathrm{m}$ fibers, this could potentially explain the large difference between the fixed and vibrating fiber stone ablation results.

In future studies, the specific direction of fiber motion without automated vibration will be studied in more detail and a force could potentially be applied parallel with this force to create a larger fiber displacement. However, this complex approach would require the forces to be synchronized in oscillation and direction to produce optimal displacement. Nevertheless, the $50-\mu \mathrm{m}$-core fiber ablation results show similar trends to that of the 100- and $150-\mu \mathrm{m}$-core fibers. The ablation volumes removed during vibrating and fixed fiber modes were similar, with the vibrating removing only $15 \%$ more volume, and the ablation depth for vibrating fiber being about the same as for fixed fiber, with greater surface area. Overall, the vibrating fiber technique appears to be consistent with a dusting methodology of ablating stones by producing shallower craters and a larger surface area.

\subsection{Study Limitations and Future Work}

There were several limitations encountered during these preliminary, feasibility studies. First, it was difficult to find a reliable method of applying the magnetic bead to the fiber with a small but reproducible profile. Future studies will focus on more sophisticated approaches to attaching the bead to the fiber.

Second, the overall size constraints of the vibrating fiber system will need to be addressed and the size of individual components reduced further before potential integration into the distal tip and working channel of a clinical ureteroscope. One approach would be to integrate the solenoid into the distal tip of the ureteroscope while allowing the optical fiber (with magnetic bean attached) to be inserted through the working channel, as is consistent with normal clinical practice. The pivot point would remain protected within the working channel while the distal fiber tip would extend a few millimeters beyond the ureteroscope itself. However, the current cross-sectional areas of both the magnetic bead and solenoid used in these preliminary studies still consume more space than available in conventional ureteroscopes. Additionally, shortening of the pivot point as well as reduction of both the magnetic bead and solenoid diameters would be desirable. The materials used for the magnetic bead will also need to be biocompatible.

Third, in addition to the overall size of the system, a significant limitation is the fiber displacement dependence on the material and diameter of the fiber. In this study, for consistency, three different diameter fibers all from the same manufacturer (and hence with similar fiber coatings/buffer/jackets) were used. However, a fiber jacket either of different thickness and/or material would change the resonant frequency. For example, during preliminary studies, a $200-\mu \mathrm{m}$-core fiber from another manufacturer (FP200ERT, Thorlabs) was tested and proved to be more rigid than the coating used on 50-, 100-, and $150-\mu \mathrm{m}$-core (Polymicro) fibers presented in this study.

Fourth, the normal vibration of these small fibers (especially, the 50- $\mu \mathrm{m}$-core fiber) during laser activation (and this dependency on pulse energy, pulse duration, and pulse rate) will also need to be studied in more detail, to understand the fundamentals of the motion and how it affects stone ablation results. This could potentially provide a scenario in which the normal fiber vibration could be enhanced by the automated vibration system.

Finally, these preliminary stone ablation studies were performed in noncontact mode to avoid potential damage to the vibrating fiber tip (e.g., from natural crevices on the stone surface or from fragmented pieces). In contact mode, fiber degradation and burnback are significant for smaller fiber diameters, and for our system, would result in a progressively shorter fiber tip and hence shorter pivot point as well, thus changing the resonant frequency of the system. In a worst-case scenario, a 
portion of the fiber tip including the magnetic bead could potentially break off during ablation, making the automated, vibrating system wholly ineffective. Thus, methods for protecting the distal fiber tip and/or reducing fiber burnback will need to be explored as well.

\section{Conclusions}

A preliminary, feasibility study was performed comparing an automated, vibrating distal fiber optic tip versus a fixed fiber during TFL lithotripsy, for potential future use in stone dusting applications. Automated, vibrating fibers with core diameters of 50,100 , and $150 \mu \mathrm{m}$ produced ablation craters in UA stones with a significantly larger surface area than for a fixed fiber while removing a statistically similar volume of stone material. With further development, an automated method of vibrating the fiber may provide a more convenient method of stone dusting during lithotripsy.

\section{Disclosures}

Nathaniel M. Fried is a consultant with IPG Medical Corporation (Marlborough, Massachusetts), Monteris Medical (Plymouth, Minnesota), and Elite Medical Experts (Tucson, Arizona). He does not hold any financial interests in these companies.

\section{Acknowledgments}

The authors thank Nicholas Giglio and Luke Hardy for their help with these studies. A part of the material presented in this paper has been previously published as an SPIE conference proceedings paper [L. A. Hall, D. A. Gonzalez, and N. M. Fried, "Thulium fiber laser stone dusting using an automated, vibrating optical fiber," Proc. SPIE 10852, 108520C (2019)].

\section{References}

1. C. D. Scales et al., "Prevalence of kidney stones in the United States," Eur. Urol. 62(1), 160-165 (2012).

2. V. Romero, H. Akpinar, and D. Assimos, "Kidney stones: a global picture of prevalence, incidence, and associated risk factors," Rev. Urol. 12(2-3), e86-e96 (2010).

3. C. Yang, S. Li, and Y. Cui, "Comparison of YAG laser lithotripsy and extracorporeal shock wave lithotripsy in treatment of ureteral calculi: a meta-analysis," Urol. Int. 98(4), 373-381 (2017).

4. J. S. Lam, T. D. Greene, and M. Gupta, "Treatment of proximal ureteral calculi: holmium:YAG laser ureterolithotripsy versus extracorporeal shock wave lithotripsy," J. Urol. 167(5), 1972-1976 (2002).

5. J. Tracey et al., "Ureteroscopic high-frequency dusting utilizing a 120-W holmium laser," J. Endourol. 32(4), 290-295 (2018)

6. S. L. Hecht and J. S. Wolf, "Techniques for holmium laser lithotripsy of intrarenal calculi," Urology 81(2), 442-445 (2013).

7. C. A. Dauw et al., "Contemporary practice patterns of flexible ureteroscopy for treating renal stones: results of a worldwide survey," J. Endourol. 29(11), 1221-1230 (2015).

8. B. R. Matlaga et al., "Ureteroscopic laser lithotripsy: a review of dusting vs fragmentation with extraction," J. Endourol. 32(1), 1-6 (2018).
9. E. J. Min et al., "Two-dimensional scanning probe driven by a solenoid based single actuator for optical coherence tomography," Opt. Lett. 36(11), 1963-1965 (2011).

10. E. J. Min et al., "Single-body lensed-fiber scanning probe actuated by magnetic force for optical imaging," Opt Lett. 34(12), 1897-1899 (2009).

11. C. M. Gridley and B. E. Knudsen, "Digital ureteroscopes: technology update," Res. Rep. Urol. 9, 19-25 (2017).

12. E. J. Seibel and Q. Y. J. Smithwick, "Unique feature of optical scanning, single fiber endoscopy," Lasers Surg. Med. 30(3), 177-183 (2002).

13. B. G. Saar et al., "Coherent Raman scanning fiber endoscopy," Opt. Lett. 36(13), 2396-2398 (2011).

14. C. M. Lee et al., "Scanning fiber endoscopy with highly flexible, 1-mm catheterscopes for wide-field, full-color imaging," J. Biophotonics 3(5-6), 385-407 (2010).

15. N. M. Fried, "Recent advances in infrared laser lithotripsy," Biomed. Opt. Express 9(9), 4552-4568 (2018).

16. N. M. Fried and P. B. Irby, "Advances in laser technology and fiberoptic delivery systems in lithotripsy," Nat. Rev. Urol. 15(9), 563-573 (2018).

17. N. J. Scott, C. M. Cilip, and N. M. Fried, "Thulium fiber laser ablation of urinary stones through small-core optical fibers," IEEE J. Sel. Top. Quantum Electron. 15(2), 435-440 (2009).

18. R. L. Blackmon, P. B. Irby, and N. M. Fried, "Thulium fiber laser lithotripsy using tapered fibers," Lasers Surg. Med. 42(1), 45-50 (2010).

19. R. L. Blackmon et al., "Thulium fiber laser ablation of kidney stones using a 50- $\mu \mathrm{m}$-core silica optical fiber," Opt. Eng. 54(1), 011004 (2015).

20. D. J. Griffiths, Introduction to Electrodynamics, Cambridge University Press, Cambridge, UK (2018).

21. J. R. Taylor, Classical Mechanics, University Science Books, Sausalito, California (2005).

22. R. L. Blackmon, P. B. Irby, and N. M. Fried, "Enhanced thulium fiber laser lithotripsy using micro-pulse train modulation," J. Biomed. Opt. 17(2), 028002 (2012).

23. H. Lee et al., "Urinary calculus fragmentation during Ho:YAG and Er: YAG lithotripsy," Lasers Surg. Med. 38(1), 39-51 (2006).

24. R. Sroka et al., "Impact of pulse duration on Ho:YAG laser lithotripsy: treatment aspects on the single-pulse level." World J. Urol. 33, 479-485 (2015).

25. F. Pigeon et al., "Optical fibre Young modulus measurement using an optical method," Electron. Lett. 28(11), 1034-1035 (1992).

26. M. Yasin, S. W. Harun, and H. Arof, Selected Topics on Optical Fiber Technology, InTech, London (2012).

27. P. Kronenberg and O. Traxer, "In vitro fragmentation efficiency of holmium:yttrium-aluminum-garnet (YAG) laser lithotripsy-a comprehensive study encompassing different frequencies, pulse energies, total power levels and laser fibre diameters," BJU Int. 114(12), 261-267 (2014).

Layton A. Hall is currently a PhD student in the Optical Science and Engineering program at the University of North Carolina at Charlotte.

David A. Gonzalez is currently a PhD student in the Optical Science and Engineering Program at the University of North Carolina at Charlotte.

Nathaniel M. Fried, PhD, is a professor in the Department of Physics and Optical Science, the University of North Carolina at Charlotte. He also holds adjunct faculty positions in the McKay Department of Urology, Carolinas Medical Center and the Department of Urology, Johns Hopkins University. His research interests include therapeutic and diagnostic applications of lasers in urology. 